\title{
Elements of Augmented Reality for Vision Systems
}

\author{
M. PiszczeK*, M. Zarzycki And M. Szustakowski \\ Institute of Optoelectronics, Military University of Technology, S. Kaliskiego 2, 00-908 Warsaw, Poland \\ The purpose of this article is a discussion about the ongoing research at the Institute of Optoelectronics, \\ Military University of Technology. The aim of the research is obtaining the introduction augmented reality \\ elements in vision systems. Technology of augmented reality in connecting with applied sensor sets can strengthen \\ and expand our sensory modalities. The process of data acquisition and processing can be implemented using \\ variable technologies, appropriate for application. At the conclusion we briefly presented two applications currently \\ under tests.
}

PACS: 42.30.Sy, 42.30.Tz, 87.63.L-

\section{Introduction}

Human perceptual capabilities to obtain information are set around several senses, which nature has endowed us. We use mainly the sight, but other senses are valuable source of information. Evolutionary success of Homo sapiens is not based on the uniqueness of our senses, but on the skills of processing them. We are aware of limitations of our sensory capabilities both in terms of sensitivity and inability to detect specific sensory modality (e.g. magnetic field). Also advanced skills in data processing sometimes appear to lead to incorrect analysis (e.g. visual illusions). With the progress of civilization, a man gradually develops series of "technical and technological prostheses" in order to eliminate our variable evolutionary constraints. Telescopes and microscopes allow us to reach into the macro and micro world. Computerized tomography or magnetic resonance imaging allows us to look inside the human body. The subject of this study is a very interesting technology called augment reality [1-5]. A characteristic feature of this solution is the ability to make information available to us via video channel, which are not directly accessible to our senses, such as location of an unknown object, which we cannot see. The process of data acquisition can be implemented using variable technologies, while the visualization of the information is a result of the computer data synthesis in the egocentric coordinate system.

\section{Machine vision and augment reality}

The machine vision is a cooperation system between electronic and optoelectronic devices which has a primary function that is an automatic analysis field of view in similar way as a human eyesight.

\footnotetext{
* corresponding author; e-mail: mpiszczek@wat.edu.pl
}

Such systems usually consist of three basic elements [1-4]:

— data logging device (image acquisition),

- data processing device,

- main processing unit, providing processed information.

Due to the nature of components and processor's capacity machine vision systems are distinguished as follows [5-7]:

a) soft sensors - consisting of a camera, a microprocessor, and sometimes an integrated illumination in a single enclosure. The sensors of this type are used in cameras with a relatively low resolution (up to $640 \times 480$ pixels - VGA) with low capacity processors [7]. Primary use of vision sensors includes reading codes, simple measurements, identifying the presence of objects, etc.

b) smart camera (type NI 1764) - consists of a camera sensor integrated with an embedded system into one housing. Cameras of this type have a high resolution (up to $2448 \times 2050$ pixels $-5 \mathrm{MP}$ ) and more accurate measurement potential. They have an operating system on board, and they can be easily programmed. Smart cameras are used for more advanced applications and they usually require high speed and high processing capacity.

c) set of camera sensor and a dedicated PC - is a system composed of a camera or multiple cameras working with a physically separate computer such as PC. This configuration allows selecting any type of camera, optimized for design application (Fig. 1).

Regardless of the type, the key role of the vision system is the ability to obtain information which are the basis to take a decision. Machine vision systems are often encountered as industrial applications that are used for example to check the physical characteristics of objects, such as size, shape, color, surface condition (roughness, printing, 


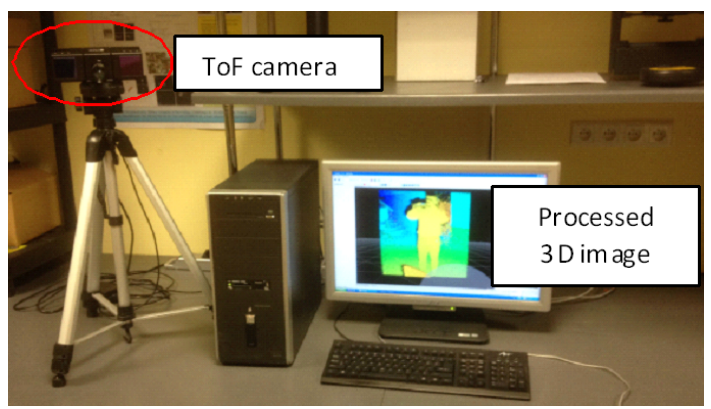

Fig. 1. Set of ToF camera and dedicated PC, PMD[vision] ${ }^{\circledR}$ CamCube 3.0.

etc.). For example, in the production process, the data obtained by the vision system usually is used by industrial robots. Information on the performance is delivered to automation controllers, for example to control their drivers. Currently, industrial vision systems are usually used to control product quality.

From the above brief analysis of vision systems, the following two-key-issues appear:

a) the vision system acquires image data and analyzes them to extract relevant information for currently running process,

b) the obtained information is a basis for decision-making process and effective action.

Confronting, in this case of the vision system with augmented reality (AR), you could say that augmented reality is a way of combining collected data (using sensors) directly from the environment of computer-generated data to provide synthesized information to the user. However, you need to consider two aspects: technological and psychological, to be able to implement effectively elements of augmented reality for vision. Cognitive psychology provides us with valuable information. They explain not only the importance of vision for human existence and his functions, but also the importance of image information resulting from the interaction of different sensory modalities. There is a particularly interesting issue of combining: visual data with data from kinesthetic sense and skills that are learnt. In terms of technology, we can compare it to the fusion of data from the image sensor, sensor orientation in $3 \mathrm{D}$ space and computer memory (Fig. 2).

How do I show the possibilities of implementation of AR which apply to above analysis?

You need to consider the following issues:

a) the user of the system,

b) fusion of sensor data and the data generated by computer.

The AR system user is basically a human being endowed with a number of senses, along with advanced data processing ability of different sensory modalities. Focusing even only on the visual modality we see that the technical equipment including camera systems allows us to reach where the eye does not reach. It may refer to the

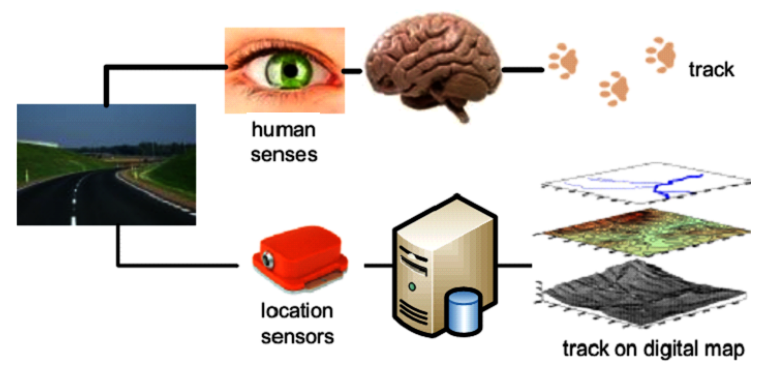

Fig. 2. Comparison of human and digital tracking system.

scale of the observed phenomena but also to the speed with which they run. The problem is not just the data acquisition but also a number of analytical issues. Realization of some of them might be far more effective by humans than machines and in other cases - completely opposite. Building video devices is a support for human activity that is primarily aimed at "sparing" him in those areas where technology is just more effective:

- restrictions on the possible observation of distant objects can be done using such cameras with telephoto lenses;

— for counting fast moving objects in a field with a limited view using e.g. linear cameras, etc.
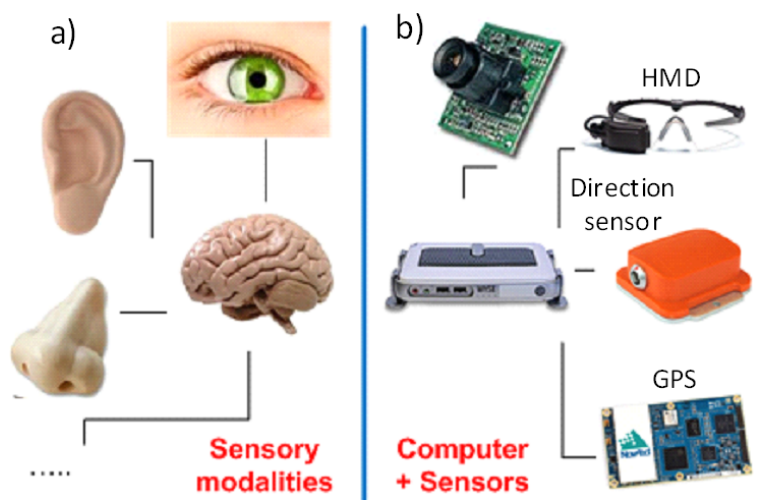

Fig. 3. Mechanisms of link data in the cognitive psychology and technological aspect.

The main aim of vision systems that support human activity is the acquisition and processing of image data under specific conditions for observing and measuring the well-defined objects of research. The man often does not have skills such as advanced vision systems. However, it is reflected in the need to cope in completely new situations in which the importance of the ability to combine data from different sensory modalities is required. Here comes the key issue of this article - the fusion of data. It is basically applicability of solutions for augmented reality. Imagine an analytical and sensory human system (Fig. 3a) and confront it with the classic system of 
video camera-computer and its expanded configuration with additional sensor elements (Fig. 3b).

By providing a vision system in any sensor, there is an increase of its operational capacity and thus the potential for information (e.g. use of GPS allows geocoding of data).

Using additional telecommunications solutions available today we get the opportunity to develop mobile video system information, which is able to support human activities. The general idea of this solution is shown in Fig. 4.

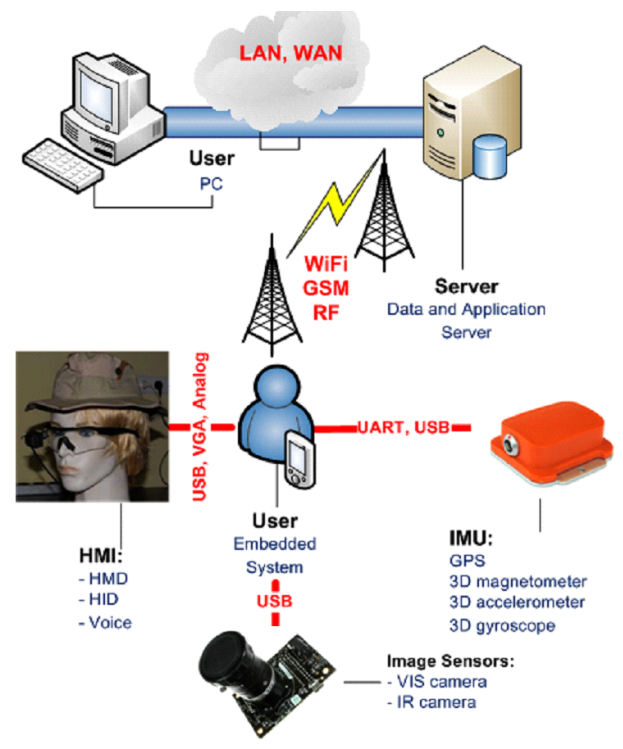

Fig. 4. Mobile AR Vision system.

This is how components can be used to build such a system that depends on the needs of a particular application and the creativity of the designer.

\section{Hardware components of information vision system}

Research that was carried out recently focuses on the selection of equipment that allows you to configure the position of augmented reality research.

The basic elements are:

a) devices for viewing,

b) the spatial location of sensors,

c) determining the spatial orientation sensors,

d) control modules and data processing.

In previous tests, data visualization are used (except traditional monitors) integrated-type HMD (head-mounted display) versions, monocular HMD and binocular HMD.

a) Vuzix iWear VR920. Main parameters: see-through - NO, display type - LCD, display resolution - VGA $(640 \times 480)$, video input - VGA.

b) Vuzix Tac-Eye LT. Main parameters: see-through - NO, display type - AMOLED, display resolution $\mathrm{SVGA}+(852 \times 600)$, video input $-\mathrm{VGA}$. c) LASTER Pro Mobile Display (Fig. 5). Main parameters: see-through — YES, display resolution — SVGA $(800 \times 600)$, video input - VGA.

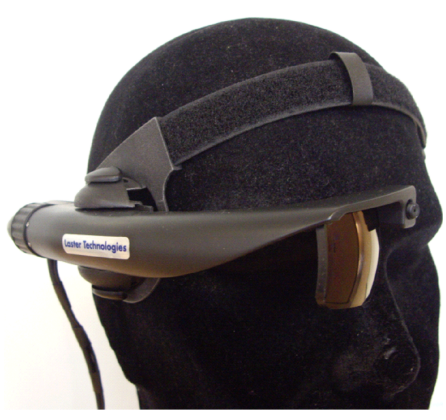

Fig. 5. LASTER Pro Mobile Display.

The first standard models GPS (e.g. Gamin GPS 18 $5 \mathrm{~Hz}$ ) were used for spatial location. To obtain more exact measures, Novatel OEM628 model was used in a research on restricted area.

OEM628 is low-power 120 channel GPS receiver, which tracks all current and upcoming satellite signals including GPS, GLONASS, Compass and Galileo. Module uses technique RT-2 to achieve $1 \mathrm{~cm}+1 \mathrm{ppm}$ horizontal position accuracy (RMS).

The Earth relation tracking (ERT) device like XSENS Mti-G was used to determine spatial orientation. It contains accelerometer, gyroscope, magnetometer and GPS. Such device is assigned as a unit of inertial measurement unit (IMU). There is a built-in microprocessor that requires a low-level of energy usage. It delivers information in $3 \mathrm{D}$ format with frequency of $120-512 \mathrm{~Hz}$ and calibrated data that applies to speed of $3 \mathrm{D}$.

Testing of sensor models (in different configurations) were made using a portable computer.

The Wyse S10 embedded PC was suggested as a central unit for building mobile applications of augmented reality. Its features are $\mathrm{H} \times \mathrm{W} \times \mathrm{D}\left(34 \times 177 \times 121 \mathrm{~mm}^{3}\right)$ with a built-in Windows XP operating system [7].

\section{Solutions applicability}

Applicability of vision systems that use the elements of augmented reality could be very wide spread. In MUT there have been projects carried out recently that aim to implement the augmented reality solutions.

In a project "Integrated system of laser photography for monitoring opened spaces and preventing terrorist threats", the AR technology has been used directly to technically advanced camera called machines of laser photography. Unique features of this camera make the user being able to define $3 \mathrm{D}$ observation area and even more - to measure linear objects.

Using sensor elements (navigation) and communication will help the operator to cooperate with other dispread elements of the system. That will improve the process of looking for objects e.g. that are found by radar system. The interface navigation model is shown in Fig. 6 . 


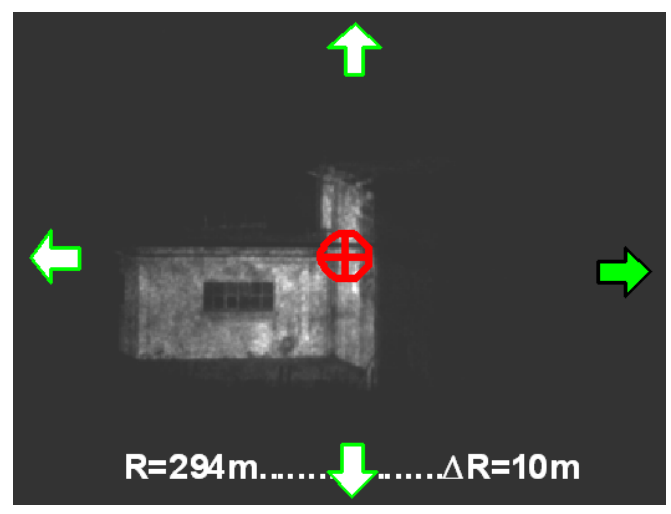

Fig. 6. Navigation user interface of UFL.

In a project "Integrated system of local antiterrorist defence" it was suggested to use data from vision monitoring system that allows to position objects in coordinates of interested field. Automatic distribution of information to operating services in the area could be made in an augmented-reality-mode (Fig. 7).

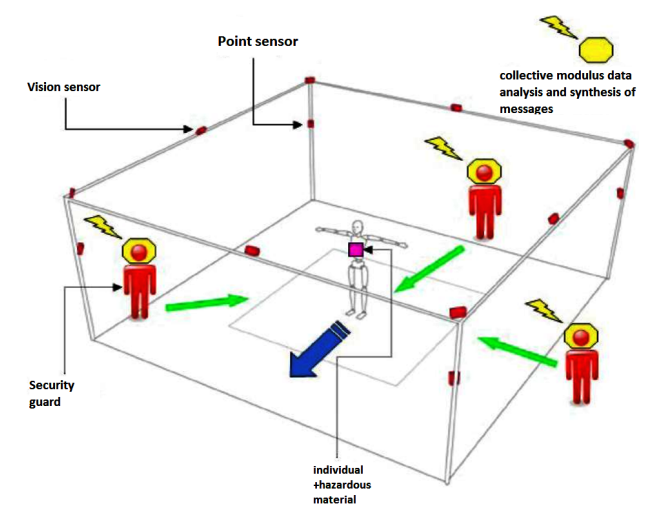

Fig. 7. Concept of using AR in defence services operations.

\section{Summary}

The vision systems supported with sensor information strongly increase their analytic possibilities. Implementation of elements of augmented reality in those systems gives users a wide range of new functional solutions in navigation and spatial information. The presented concepts of using technology AR directed with machines of laser photography and with systems do support defence service operations is only one from many other possible implementations. This technology can be used in a wide range of other areas, e.g. medicine, navigation, industry. With the progressive miniaturisation of sensors, the increase of power capacity portable computers and access to portable machines of visualisation, the AR implementation is rapidly growing and the only one restrain to it so far, seems to be a human creativity.

\section{Acknowledgments}

This work was founded by the Polish Ministry of Science and Higher Education (project OR00000312).

\section{References}

[1] P. Pardel, Studia Informat. 30, 35 (2009).

[2] M. Piszczek, Acta Phys. Pol. A 120, 716 (2011).

[3] P. Milgram, H. Takemura, A. Utsumi, F. Kishimo, Proc. SPIE Telemanipulator Telepresence Technol. 6351, 34 (2007).

[4] T. Pustelny, J. Ignac-Nowicka, B. Jarzabek, A. Burian, Opt. Appl. 34, 551 (2004).

[5] K. Gut, D. Nabaglo, Acta Phys. Pol. A 116, 307 (2009).

[6] E. Nęcka, J. Orzechowski, B. Szymura, Cognitive Psychology, PWN: SWPS "Academica", Warsaw 2008 (in Polish).

[7] http://industrial.omron.pl . 